\title{
Emotional Abuse in Sport: A Case Study of Trichotillomania in a Prepubescent Female Gymnast
}

\author{
Misia Gervis* and Richard Godfrey \\ Brunel Centre for Sport, Health and Wellbeing, Middlesex, United Kingdom
}

\begin{abstract}
Despite improved legislation in most countries, child abuse in sport continues to exist but is a problem which is often under reported or ignored. In elite sport 'suffering' is not uncommon and hence sometimes child abuse is sometimes unrecognised, de-emphasised or easily dismissed as part of a collective experience that is perceived to be necessary to 'create' elite athletes. However, even swearing, anger, raised voices and negative comments directed at child athletes by coaches is considered abuse and can, when regular and routine, cause long term wellbeing and health issues. Self-harm can be a consequence and here self-harm in the form of trichotillomania, self hair-pulling, is reported for the first time as a secondary consequence of abuse. The 12 year old female gymnast, subject of this case study, presented with this impulse control disorder as defined by the American Psychiatric Association and was successfully treated using cognitive behavioural therapy. However, the training environment, including coach behaviour, did not change and so the gymnast remained at risk of recurrence of self-harm. Such environments in sport have many characteristics in common with and reminiscent of religious cults; sacrifice, isolation, shared obsession, a charismatic leader, and often in the presence of severe calorie restriction. As a consequence of ageing, growth, injury and an unchanging abusive environment, a year later the gymnast retired from the sport.
\end{abstract}

\section{Introduction}

In recent years more research has exposed incidences of child abuse in sport and particularly on that perpetrated by sports coaches. Abuse enacted by coaches on their athletes has been shown to occur in many forms; physical [1], psychological [1], emotional [2] and sexual [3].

Sexual abuse and harassment in sport has been widely reported and there is now a body of research that highlights the vulnerability of athletes to sexual abuse and in particular athletes competing at the elite levels of sport [4-7]. Indeed the most recent reported case of sexual abuse in sport has emerged from Penn State University where the head football coach was found guilty of 45 counts of sexual abuse in November 2012.

Emotional abuse is also emerging as a cause for concern with many normalized coaching practices being exposed as fundamentally emotionally abusive and psychologically damaging to young elite athletes [2,8-10].

In particular, with reference to elite female gymnastics, Tofler et al. [11] reported that: "In general, they [elite gymnasts] are in awe of their coaches and other adult authorities who hold the key to their potential success; consequently, they are at risk for abuse". Indeed it has been further reported that the relationship between gymnastic coaches and their gymnasts is like master and slave [12]. Training under these conditions affords the gymnast no power to voice their emotional, psychological and physical needs. This further supports the notion that young gymnasts are particularly vulnerable to being abused by their coaches.

In the UK, sexual abuse by sports coaches has received a lot of attention since the first cases emerged in the 1990s [13,14] and this has led to improved child protection policies enacted in the UK. Despite a large number of UK sports governing bodies, including the British Gymnastics Association (BGA), signing-up to child protection and instigating their own policies, emotional abuse of all types still seems to occur to gymnasts in some gyms and indeed this seems true across a variety of sports in the UK.

The culture of compliance that seems to exist between an athlete and his or her coach is so prevalent in elite sport that there is an increased danger where vulnerability is heightened and self worth is lessened [15].
As a consequence the coach is in a position of considerable influence which subjects the elite child athlete to great risk should this power be misused. In reviewing the sociological literature on the child athletecoach relationship, Burke [16], highlighted the power that the coach had over the child athlete and stated:

"Coaches often view their athletes as their possessions. They are wary of outside judgement and questioning of their tactics, philosophies and practices in coaching. They may enact any number of restrictions on their charges, restrictions that are [normally] only placed by parents."

The consequences of abuse are varied but are generally first seen as an increase in anxiety and a decrease in self-esteem. This latter factor often marking a cascade towards more severe clinical symptoms such as depression [17], obsessive-compulsive behaviours such as impulse control disorders and eating disorders [18]. Prolonged abuse, even with a good coping mechanism or strategy, can result in impaired personality development and the appearance of personality disorders as an adult [19]. Further, prolonged abuse can sufficiently negatively impact self-esteem and self-worth to engender thoughts of suicide which in a few extreme cases can be realised and result in fatality [20].

Perhaps it is logical to assume that 'high profile' abuse such as sexual violation and physical assault can lead to long term psychological dysfunction. However, less obvious emotional abuse such as 'frequent and/or repetitive mild acts' of emotional abuse could also result in a negative outcome and further, perhaps there is a continuum of aversion

*Corresponding author: Misia Gervis, PhD, Brunel Centre for Sport, Health and Wellbeing, Heinz Wolff Building, Kingston lane, Uxbridge, Middlesex, United Kingdom, UB8 3PH, E-mail: misia.gervis@brunel.ac.uk

Received December 12, 2012; Accepted March 21, 2013; Published March 23 2013

Citation: Gervis M, Godfrey R (2013) Emotional Abuse in Sport: A Case Study of Trichotillomania in a Prepubescent Female Gymnast. J Clin Case Rep 3: 264 doi:10.4172/2165-7920.1000264

Copyright: ( 2013 Gervis M, et al. This is an open-access article distributed unde the terms of the Creative Commons Attribution License, which permits unrestricted use, distribution, and reproduction in any medium, provided the original author and source are credited. 
from mild to severe which may cause 'irreversible damage' [21]. This is especially true when abusive behaviour is directed at a child by a significant adult [22].

Of the varied consequences of emotional abuse in sport, trichotillomania has never before been documented. The term 'trichotillomania' was first coined by the French nineteenth century physician François Henri Hallopeau and refers to the repetitive pulling out by the patient, of their own hair. The Diagnostic and Statistical Manual of Mental Disorders (DSM-IV) classifies it as an impulse control disorder [23].

\section{Case Presentation}

A 12-year old elite female gymnast with 5 years substantial training experience (20 hours per week from age 6-9 yrs and 30 hours per week from age $9 \mathrm{yrs}$ ) and who competes at elite level is the subject of this case study. The patient presented with bald patches on the head and on interview was found to fulfil DSM-IV criteria for trichotillomania. That is "recurrent pulling out of one's own hair that results in noticeable hair loss", "increasing sense of tension immediately before pulling out of hair", pleasure, gratification or relief when pulling out the hair" [23]. Although trichotillomania can exist without all of these criteria being met, in the current case study all of the above criteria were present. Currently there is no 'gold standard' for assessment of trichotillomania and so assessment relies on a number of methods including selfmonitoring and self-reporting. In addition, a 7-point summative response scale to rate hair loss has been devised by Diefenbach et al. [23]. This rates severity from 1-no evidence of hair pulling to 7-large bald spots and on this scale, the psychologist and patient agreed a rating of 6.

From the age of 9 yrs the subject became a weekly border at a school in England to facilitate her training at her new elite gymnastics club. Anxiety increased steadily until six months later she was regularly pulling out her own hair. As a result of school and parental concern the help of a psychologist was sought. Through therapy it was determined that the hair pulling occurred when the client was overly fatigued and overly anxious. It was found to be an 'unconscious' process, but relief was experienced following the behaviour. The case presents a number of potential confounding factors that may have contributed to her elevated anxiety state. However, the child reported to be happy at school and at home, indicating that she felt supported by her family and by school staff, thus these were ruled out as major contributing factors. Therefore, the training environment of the club was examined and emotionally abusive behaviour by coaches directed at young gymnasts was found to be the norm. This included belittling, public humiliation, shouting and generally aggressive and intimidating behaviour.

Coaches provided significantly more negative reinforcement with positive reinforcement coming only from fellow female gymnasts who were part of the 'tightly' cohesive group of athletes that had evolved in this environment.

\section{Treatment}

The treatment programme implemented with this patient was, essentially, anchored in a Cognitive Behaviour Therapy (CBT) approach. The therapeutic intervention was conducted over a period of four months on a weekly and then biweekly basis. Whilst the majority of work was done directly with the patient, observations were also made of the training environment, and lengthy discussions were had with both the parents and coaches. The initial work focused on understanding the instances, intensity, and triggers of the hair pulling behaviour. When gently encouraged to examine her own head she acknowledged that she did not like the appearance and that it made her ashamed, self-conscious and embarrassed.

The psychologist and athlete together identified stages or landmarks in the build-up to hair-pulling that allow the athlete to recognise and use diversionary behaviour to prevent, or short-circuit, the course of events that ultimately result in hair being pulled out. This was achieved through a combination of interventions to help manage the anxiety including; relaxation (breath control); thought stopping; positive self-talk and imagery. This approach was successful in significantly diminishing the hair pulling behaviour, and giving the patient anxiety management skills, but some concerns remained. Through the therapy it became apparent that the patient was encased in an environment where coaches demanded complete compliance and a subservient demeanour. Given the amount of time that she spent in this environment with very little time with her family, she learnt acceptance of this emotionally damaging culture as being 'normal'. It became evident that she had no friends outside of this environment, and no other influences on her life that might challenge the world that she lived in. Her feelings of self-worth and self-esteem were directly affected by her coaches' behaviour towards her.

\section{Discussion}

Discussion with the coaches was not fruitful as they did not concede that their behaviour was in any way responsible for the athlete's anxiety or disordered behaviour. In their view their behaviour is not abusive but rather is a part of the culture of gymnastics. Such behaviour/treatment described by the coaches as the need to 'crush' the individual, facilitates, in their opinion, ascendance to the highest levels of competition. The matter was also raised with Head of Sport Science and Medicine (British Gymnastics Association). No specific cases of trichotillomania, secondary to emotional abuse, are recorded in sport and the authors believe this to be the first documented case.

With respect to emotional abuse in sport generally there could be said to be parallel sequelae with religious cult psychology and with Complex Post Traumatic Stress Disorder (C-PTSD). Individuals who become part of a religious cult are generally already emotionally weak and or vulnerable and tend to gravitate towards shared aspirations and beliefs [24]. The gymnast, who is the subject here, is a young female who is isolated from family by being in a boarding school and only has friends in gymnastics as they are the only ones who are entirely empathetic. Cults recognise the authority of a leader or founder, there is common practice of rituals, and there is sacrifice and fear of expulsion [24]. The parallels in the abused gymnast are the unchallengeable authority of the coach and where the common practice of rituals refers to 30 hours per week of physical training. Sacrifice is seen in the lack of a social life and lack of friends outside gymnastics. Fear of expulsion is more commonly recognised in gymnastics as a fear of being unsuccessful in achieving a new physical skill, not qualifying for an event or not doing well at an event and hence being alienated from the group and includes feelings that you are despised by the coach. To reiterate, the child reported being happy both at school and at home.

This environment heightens anxiety, attacks self-worth and diminishes self-esteem. Over time repetitive abuse may result in an adult who displays signs and symptoms which are similar to those found in complex post traumatic stress disorder. C-PTSD, or disorders of extreme stress not otherwise specified (DESNOS) [25,26] is more likely to involve deficits in the capacity for self-regulation and so, such individuals do not always respond as well, or may respond adversely 
to treatment for PTSD [27]. For example, McDonagh-Coyle et al. [28] found a high attrition rate (30\%) in individuals who had suffered longterm abuse when treatment involved combined Prolonged Exposure and Cognitive Restructuring (PE/CR) versus "Present-Centred Therapy" (PCT) where there was a low (10\%) attrition rate.

A year after this situation coalesced into that reported above, the subject had a few other 'problems' which gave cause for further reflection. These included growth and development which made some technical aspects of the sport increasingly difficult and OsgoodSchlatter's disease. These, coupled with the abusive environment, ultimately culminated in the gymnast's decision to leave the sport.

\section{Conclusion}

- Trichotillomania represents another behaviour which can result as a secondary response to emotional abuse in sport and has never before been documented.

- Child abuse in sport appears to have parallels with religious cult psychology and where low level, long duration (years of) abuse is in operation, signs and symptoms of complex post traumatic stress disorder (C-PTSD) may also be apparent.

- Emotional abuse of all types continues to be directed at children in sport despite huge steps forward in recognition for and implementation of child protection policies. Children who participate in elite sport are rarely seen as vulnerable, and are certainly not considered to be 'at risk'; this assumption should be challenged.

- Emotionally abusive behaviour appears to be endemic in the culture of elite child sport and steps should be taken to confront this behaviour and enhance awareness of its destructive nature.

- Cases of child abuse in sport are not always straight forward and patients may not always respond well to conventional treatment. In such cases treating both the acute symptoms and the underlying causes are extremely important. Without treating underlying causes, the risk of further psychological damage appearing years after the event is significantly increased.

\section{References}

1. Hong F (2004) Innocence lost: Child athletes in China. Sport Soc 7: 338-354

2. Gervis M, Dunn N (2004) The emotional abuse of elite child athletes by their coaches. Child Abuse Rev 13: 215-223.

3. Brackenridge $\mathrm{CH}$ (1994) Fair play or fair game? Child sexual abuse in sport organisations. Int Rev Soc 29: 287-298.

4. Brackenridge $\mathrm{CH}$ (2001) Spoilsports. Routledge.

5. Sundgot-Borgen J, Fasting K, Brackenridge C, Torstveit MK, Berglund B (2003) Sexual harassment and eating disorders in female elite athletes-a controlled study. Scand J Med Sci Sports 13: 330-335.

6. Kirby S (2012) Sexual harassment and abuse in Canadian sport. In: Sport, Children's Rights and Violence Prevention: A Sourcebook on Global Issues and Local Programmes. Brackenridge, Kay and Rhind (Eds), Brunel University Press, UK

7. Leahy T (2012) Sexual abuse in competitive sport in Australia. In: Sport, Children's Rights and Violence Prevention: A Sourcebook on Global Issues and Local Programmes. Brackenridge, Kay and Rhind (Eds), Brunel University Press, UK.

8. Gervis M (2010) From concept to model: A new theoretical framework to understand the process of emotional abuse in elite child sport. In: Elite ChildAthlete Welfare: International Perspectives. Brackenridge and Rhind (Eds), Brunel University Press, UK.

9. Gervis M (2012) Child athletes' negative emotional responses to their coaches in the United Kingdom. In: Sport, Children's Rights and Violence Prevention: A
Sourcebook on Global Issues and Local Programmes. Brackenridge, Kay and Rhind (Eds.)., Brunel University Press, UK.

10. Stirling A, Kerr G (2012) Emotional abuse in Canadian sport. In: Sport Children's Rights and Violence Prevention: A Sourcebook on Global Issues and Local Programmes. Brackenridge, Kay and Rhind (Eds.)., Brunel University Press, UK.

11. Tofler IR, Stryer BK, Micheli LJ, Herman LR (1996) Physical and emotional problems of elite female gymnasts. N Engl J Med 335: 281-283.

12. David P (2005) Human Rights in Youth Sport: A critical review of children's rights in competitive sports. European PE Review.

13. Fasting K, Brackenridge $\mathrm{CH}$ (2009) Coaches, sexual harassment and education. Sport, Education and Society 14: 21-35

14. Downes S (2002) Every Parent's Nightmare. Observer Sport Monthly.

15. Jones RL, Glintmeyer N, McKenzie A (2005) Slim bodies, eating disorders and the coach-athlete relationship: a tale of identity creation and disruption. Int Rev Soc Sport 40: 377-391.

16. Burke M (2001) Obeying until it hurts: coach-athlete relationships. J Phil Sport 28: $227-240$

17. Bingelli NJ, Hart SN, Brassard MR (2001) Psychological maltreatment of children. Sage Publications, London.

18. Cohen P, Brown J, Smaile E (2001) Child abuse and neglect and the development of mental disorders in the general population. Dev Psychopatho 13: $981-999$.

19. McGee RA, Wolfe DA, Wilson SK (1997) Multiple maltreatment experiences and adolescent behavior problems: adolescents' perspectives. Dev Psychopathol 9: 131-149.

20. Navarre EL (1987) Psychological maltreatment: The core component of child abuse. In: Psychological maltreatment of children and youth. Brassard Germain, Hart (Eds.). Pergamon Press, New York.

21. O'Hagan KP (1995) Emotional and psychological abuse: problems of definition Child Abuse Negl 19: 449-461.

22. Marshall MB, Zuroff DC, McBride C, Bagby RM (2008) Self-criticism predicts differential response to treatment for major depression. J Clin Psychol 64: 231 244

23. Diefenbach GJ, Tolin DF, Hannan S, Maltby N, Crocetto J (2006) Group treatment for trichotillomania: behavior therapy versus supportive therapy Behav Ther 37: 353-363.

24. Chambers W, Langone M, Dole A, Grice J (1993) The Group Psychological Abuse Scale: A Measure of the Varieties of Cultic Abuse. Cultic Studies Journal 11: 88-117.

25. Herman JL (1992) Complex PTSD: A syndrome in survivors of prolonged and repeated trauma. J Traumatic Stress 5: 377-392.

26. van der Kolk BA, Pelcovitz D, Roth S, Mandel FS, McFarlane A, et al. (1996) Dissociation, somatization, and affect dysregulation: the complexity of adaptation of trauma. Am J Psychiatry 153: 83-93.

27. Ford JD (1999) Disorders of extreme stress following war-zone military trauma: associated features of posttraumatic stress disorder or comorbid but distinct syndromes? J Consult Clin Psychol 67: 3-12.

28. McDonagh-Coyle A, Friedman M, McHugo G, Ford J, Mueser K, et al. (1992) Cognitive behavioral treatment for childhood sexual abuse survivors with PTSD. Proc Int Soc Traum Stress Studies 15: 377-391.

Citation: Gervis M, Godfrey R (2013) Emotional Abuse in Sport: A Case Study of Trichotillomania in a Prepubescent Female Gymnast. J Clin Case Rep 3: 264 doi:10.4172/2165-7920.1000264 